\title{
The expanding role of bendamustine in chronic lymphocytic leukemia
}

\author{
Kruti Sheth Nair \\ Chaitra Ujjani \\ Lombardi Comprehensive Cancer \\ Center, Medstar Georgetown \\ University Hospital, Washington, \\ DC, USA
}

This article was published in the following Dove Press journal:

Blood and Lymphatic Cancer: Targets and Therapy

8 July 2015

Number of times this article has been viewed

\begin{abstract}
As the most prevalent form of adult leukemia, chronic lymphocytic leukemia (CLL) affects thousands of patients each year. Given the indolent nature of the disease, symptomatic patients frequently experience multiple relapses throughout their clinical course. Better therapeutic options are needed, particularly for the elderly population that characterizes the majority of affected patients. Bendamustine, a hybrid alkylating agent, has demonstrated remarkable activity in CLL in conjunction with a tolerable safety profile. Although historically used in relapsed and refractory disease, it has recently gained a role in the front-line setting, including younger, physically fit patients. Current investigatory efforts are focused on exploring the combination of bendamustine with novel therapies in CLL.
\end{abstract}

Keywords: chronic lymphocytic leukemia, overall survival, aspartate aminotransferase, chlorambucil, bendamustine

\section{Introduction}

Chronic lymphocytic leukemia (CLL) is the most prevalent adult leukemia in the western world; however, the incidence is relatively lower in Africa and Asia. ${ }^{1}$ The median age of diagnosis is 72 years, although up to $15 \%$ of patients are younger than 50-year-old. An accumulation of mature, yet dysfunctional, monoclonal B-lymphocytes that express CD19, CD23, CD5, and low levels of CD20 characterizes the disease. The expression of CD38 and ZAP-70 portend a poorer prognosis, as does the presence of deletion (del) 17p, del 11q, and unmutated immunoglobulin variable heavy chain (IGVH). Patients are classified according to the Rai or Binet staging systems, which are based on the presence of lymphadenopathy, hepatosplenomegaly, and cytopenias. ${ }^{2,3}$

Patients are frequently asymptomatic at diagnosis and can be monitored closely as early intervention with chemotherapy in this population does not impact overall survival (OS). Indications for treatment include persistent constitutional symptoms, progressive splenomegaly or lymphadenopathy, steroid-refractory autoimmune cytopenias, or a rapidly developing lymphocytosis. ${ }^{4}$ Choice of therapy should be made based on patient age, comorbidities, performance status, and lifestyle preferences. Until recently, standard front-line induction consisted of fludarabine-based chemoimmunotherapy, including rituximab with or without cyclophosphamide (FCR, FR) ${ }^{5-7}$ Although impressive overall response rates (ORRs) of $>90 \%$ have been demonstrated with these regimens, patients inevitably relapse. As allogeneic stem cell transplantation is the only curative option for this disease, CLL remains an ongoing therapeutic challenge.

Correspondence: Chaitra Ujjani Lombardi Comprehensive Cancer

Center, Medstar Georgetown University Hospital, 3800 Reservoir Road NW,

Washington, DC 20007, USA

Tel + I 2024440596

Fax + I 2024449429

Email csu@georgetown.edu 
Bendamustine is a unique chemotherapeutic agent created in the early 1960 s to provide a more tolerable option to the alkylating agents available at the time. Developed in East Germany by Ozegowski and Krebs, bendamustine's promise in the lymphoid malignancies was unknown to the rest of the world until the early 2000 s. ${ }^{8}$ It has demonstrated impressive activity, particularly in CLL, both as a single agent as well as in combination with immunotherapy. Given its efficacy and tolerability, bendamustine serves as an attractive backbone for newer biologic molecules as well. This article will review the current and future role of bendamustine in the treatment of CLL.

\section{Pharmacology}

Bendamustine is considered to be a bifunctional agent as it possesses both alkylating and antimetabolite properties. Its chemical structure consists of a 2-chloroethylamine alkylating group, a benzimidazole ring, and a butyric acid side chain (Figure 1). ${ }^{9}$ The mechlorethamine and butyric acid groups contribute to bendamustine's alkylating properties, whereas the benzimidazole ring is theorized to provide the antimetabolite activity. Given the dual features, bendamustine exhibits only partial cross-resistance to other alkylating agents. ${ }^{10}$ Its mechanisms of action include inhibition of mitotic checkpoints, enhancement of apoptosis, induction of mitotic catastrophe, and activation of DNA damage stress response and base excision DNA repair mechanisms.

Bendamustine is administered intravenously over 30-60 minutes. ${ }^{11,12}$ The half-life of bendamustine is rapid at 38-49 minutes with a mean volume distribution of $14.2-18.3 \mathrm{~L} / \mathrm{min}$. Bendamustine is primarily eliminated via feces, less than $10 \%$ is excreted in the urine. Bendamustine undergoes extensive first-pass metabolism via CYP1A2catalyzed N-dealkylation and gamma hydroxylation. ${ }^{13}$ The resulting metabolites include gamma-hydroxy-bendamustine (M3), which retains an activity level similar to the parent compound and the relatively inactive $N$-desmethyl-bendamustine (M4). Phase II metabolism consists of conjugation with gluthathione and may also play a role in the elimination of bendamustine as demonstrated in cholangiocarcinoma. ${ }^{14}$ Bendamustine can be safely administered to patients with mild-to-moderate renal failure without significantly changing

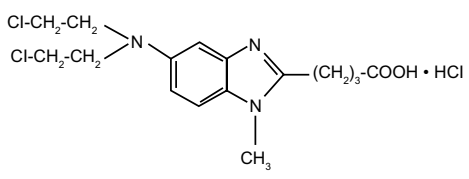

Figure I Chemical structure of bendamustine. Note: Data from Cephalon. ${ }^{\prime \prime}$ the degree of systemic exposure. ${ }^{15}$ Although it is not approved in patients with a creatinine clearance $(\mathrm{CrCl})<40 \mathrm{~mL} / \mathrm{min}$, case reports have indicated tolerability in those undergoing hemodialysis. ${ }^{16,17}$ Similarly, it can be administered to patients with mild hepatic impairment, defined as an elevated total bilirubin of 1-1.5 times the upper limit of normal or aspartate aminotransferase (AST) of 1-2.5 times the upper limit of normal. Patients with moderate or severe hepatic compromise (AST or alanine aminotransferase [ALT] $>2.5$ times the upper limit of normal or total bilirubin $>1.5$ times the upper limit of normal) may receive it safely if disease burden is felt to be the etiology of the liver dysfunction. ${ }^{18,19}$

\section{Bendamustine monotherapy Phase I/II trials}

Several Phase I and II studies have demonstrated the remarkable efficacy of bendamustine monotherapy in patients with heavily pretreated CLL (Table 1). Prior regimens consisted primarily of chlorambucil (Chl) with prednisolone, however, a small number of individuals received fludarabine, cyclophosphamide, bleomycin, or mitoxantrone. Patients achieved ORRs of $56 \%-93 \%$ with complete response (CR) rates of $7 \%-30 \%$. Bendamustine was noted to be quite tolerable, and the most common adverse events include nausea/emesis, allergic reaction, infection, and diarrhea. Notable grade $3 / 4$ toxicities were related to myelosuppression. Grade 3/4 hyperbilirubinemia was reported in $40 \%$ of patients in one study; however, this was a unique finding, which has not been demonstrated in other publications. ${ }^{20}$

\section{Phase III trials}

Based on the promising results from early investigations, bendamustine was evaluated further in a number of Phase III clinical trials. Niederle et al compared bendamustine (B) to fludarabine $(\mathrm{F})$ in relapsed and refractory patients, the majority of whom had previously received $\mathrm{Chl} .{ }^{25}$ The median age of the 92-patient cohort was 69 years. Patients were randomized to either B $100 \mathrm{mg} / \mathrm{m}^{2}$ day (D) 1 and 2 or F $25 \mathrm{mg} / \mathrm{m}^{2} \mathrm{D} 1-5$ for a maximum of eight, 28-day cycles. The results of the trial favored bendamustine in terms of ORR (76\% vs $62 \%$ ) and CR (27\% vs 9\%), although not statistically significant $(P=0.057)$. The median progression-free survival (PFS) with bendamustine was 20.1 months vs 14.8 months $(P=0.53)$, while the median OS was 44 months vs 41 months $(P=0.48)$. There were an equal number of dose reductions required in each arm $(36 \% \mathrm{~B}, 37 \% \mathrm{~F})$, as well as similar rates of grade $3 / 4$ neutropenia ( $20 \%$ vs $17 \%$ ) and infection ( $13 \%$ vs $15 \%$ ). Although in a relatively small number of patients, these were 
Table I Bendamustine monotherapy Phase I/II trials

\begin{tabular}{|c|c|c|c|c|c|c|}
\hline Author & Phase & Population & Dosing of bendamustine & ORR (CR) & Median PFS/DOR & Median OS \\
\hline Kath et $\mathrm{al}^{21}$ & II & $\begin{array}{l}\text { Untreated }(n=13) ; \\
\text { relapsed/refractory }(n=10)\end{array}$ & $60-70 \mathrm{mg} / \mathrm{m}^{2}$, days $\mathrm{I}-5$, q4 weeks & $75 \%(30 \%)$ & 7 months & I 3.6 months \\
\hline Aivado et $\mathrm{al}^{22}$ & II & Relapsed/refractory $(n=2 I)$ & $100 \mathrm{mg} / \mathrm{m}^{2}$, days I-2, q4 weeks & $67 \%(29 \%)$ & 6 months & Not reported \\
\hline Bremer $^{23}$ & II & Relapsed/refractory $(n=15)$ & $60 \mathrm{mg} / \mathrm{m}^{2}$, days $\mathrm{I}-5, \mathrm{q} 4-6$ weeks & $93 \%(7 \%)$ & Not reported & 32 months \\
\hline Bergmann et $\mathrm{a}^{24}$ & $\mathrm{I} / \mathrm{II}$ & Relapsed/refractory $(n=16)$ & $70-100 \mathrm{mg} / \mathrm{m}^{2}$, days $\mathrm{I}-2, \mathrm{q} 3-4$ weeks & $56 \%(12 \%)$ & 42.7 months & 45.6 months \\
\hline Lissitchkov et al ${ }^{20}$ & $\mathrm{I} / \mathrm{II}$ & $\begin{array}{l}\text { Relapsed/refractory, } \\
\text { fludarabine naive }(n=15)\end{array}$ & $100-120 \mathrm{mg} / \mathrm{m}^{2}$, days $\mathrm{I}-2$, q3 weeks & $60 \%(27 \%)$ & $22+$ months & Not reported \\
\hline
\end{tabular}

Abbreviations: ORR, overall response rate; CR, complete response; PFS, progression free survival; DOR, duration of response; OS, overall survival.

the first Phase III data to suggest the efficacy of bendamustine in comparison to fludarabine in the treatment of CLL.

Knauf et al conducted the first front-line Phase III trial in which bendamustine was compared directly to $\mathrm{Chl} .^{26}$ Three hundred and nineteen patients were randomized to receive either B $100 \mathrm{mg} / \mathrm{m}^{2} \mathrm{D} 1$ and 2 or Chl $0.8 \mathrm{mg} / \mathrm{kg}$ D1 and 8 for a maximum of six, 28-day cycles. Eligible patients were $<76$ years and had Binet stage $\mathrm{B}$ or $\mathrm{C}$ disease. Bendamustine was found to have a significantly higher ORR (68\% vs $31 \%)$, CR rate $(31 \%$ vs $2 \%)$, and median PFS (21.6 months vs 8.3 months) than Chl $(P<0.0001)$. The median OS among all patients favored bendamustine (not reached vs 79 months, $P=0.18$ ) and was found to be significantly higher for patients who had achieved a CR (not reached vs 75.9 months; $P=0.0018) .{ }^{27}$ Additionally, global health and quality of life scores for physical, emotional, social, and cognitive functioning were similar among the arms. Based on these data, bendamustine was approved by the
US Food and Drug Administration for previously untreated patients with CLL in 2008.

\section{Bendamustine in combination Bendamustine and rituximab}

While bendamustine has been investigated with a number of chemotherapeutics, perhaps the most successful combination thus far has been with rituximab (BR) (Table 2). Based on preclinical models demonstrating synergy and promising Phases II and III results in indolent non-Hodgkin lymphomas (NHL), the German CLL Study Group (GCLLSG) conducted a Phase II study of BR in relapsed and refractory CLL. ${ }^{28-30}$ Of the 78 patients enrolled, $81 \%$ had received prior fludarabine and $28 \%$ were fludarabine refractory. Patients received B $70 \mathrm{mg} /$ $\mathrm{m}^{2}$ cycle (C)1-6D 1 and 2 and $\mathrm{R} 375 \mathrm{mg} / \mathrm{m}^{2}$ on D0 of C1 and $500 \mathrm{mg} / \mathrm{m}^{2}$ on C2-6D1. The ORR was $59 \%$ (CR 9\%), whereas the median PFS and OS were 14.7 months and 34 months, respectively. The regimen appeared to have activity in patients

Table 2 Targeted therapies in combination with bendamustine

\begin{tabular}{|c|c|c|c|c|c|c|c|c|}
\hline Author & Phase & $\mathbf{n}$ & Disease status & Regimen & $\begin{array}{l}\text { Target of novel } \\
\text { agent }\end{array}$ & $\begin{array}{l}\text { ORR } \\
\text { (CR) }\end{array}$ & $\begin{array}{l}\text { Median } \\
\text { PFS }\end{array}$ & Median OS \\
\hline Fischer et $\mathrm{al}^{30}$ & II & 78 & Relapsed/refractory & Rituximab + B & CD20 & $59 \%(9 \%)$ & 14.7 months & 34 months \\
\hline Eichhorst et $\mathrm{al}^{32}$ & III & 273 & Untreated & Rituximab + B & CD20 & $98 \%(32 \%)$ & 43.2 months & $92 \%$ (3 years) \\
\hline Ujjani et $\mathrm{al}^{36}$ & II & 10 & Relapsed/refractory & Ofatumumab + B & CD20 & $40 \%(20 \%)$ & 8.I months & 16.2 months \\
\hline Cortelezzi et $\mathrm{al}^{37}$ & II & 47 & Relapsed/refractory & Ofatumumab + B & CD20 & $72 \%(17 \%)$ & $50 \%$ (2 years) & $84 \%$ (2 years) \\
\hline Offner et $\mathrm{al}^{38}$ & II & 44 & Untreated & Ofatumumab + B & CD20 & $95 \%(43 \%)$ & NA & NA \\
\hline Offner et $\mathrm{al}^{38}$ & II & 53 & Relapsed/refractory & Ofatumumab + B & CD20 & $74 \%(11 \%)$ & NA & NA \\
\hline Brown et $\mathrm{al}^{42}$ & $\mathrm{lb}$ & 41 & Untreated & Obinutuzumab + B & CD20 & $90 \%(20 \%)$ & NA & NA \\
\hline Gladstone et $\mathrm{al}^{48}$ & II & 50 & Relapsed/refractory & MEDI-55I + B & CDI9 & $48 \%-64 \%$ & NA & NA \\
\hline Robak et $\mathrm{al}^{49}$ & II & 65 & Relapsed/refractory & Otlertuzumab + B & CD37 & $69 \%(9 \%)$ & 14 months & NA \\
\hline Ujjani et $a^{54}$ & I & 7 & Relapsed/refractory & Lenalidomide + B & $\begin{array}{l}\text { NF- } \kappa B \text { in B-cells, } \\
\text { T-cells, NK-cells }\end{array}$ & $57 \%(14 \%)$ & 14 months & NA \\
\hline Abramson et $\mathrm{al}^{55}$ & I & 23 & Untreated & Lenalidomide + BR & $\begin{array}{l}\mathrm{NF}-\kappa \mathrm{B} \text { in B-cells, } \\
\mathrm{T} \text {-cells, NK-cells }\end{array}$ & $87 \%(38 \%)$ & NA & NA \\
\hline Brown et $\mathrm{al}^{59}$ & III & 289 & Relapsed/refractory & Ibrutinib + BR & BTK & $83 \%(10 \%)$ & Not reached & Not reached \\
\hline Barrientos et $\mathrm{al}^{62}$ & $\mathrm{I}$ & 50 & Relapsed/refractory & Idelalisib + BR, B or R & PI3K delta & $81 \%(2 \%)$ & $62 \%$ (2 years) & $85 \%$ (2 years) \\
\hline Flinn et $\mathrm{al}^{64}$ & $\mathrm{lb}$ & 21 & Relapsed/refractory & $\begin{array}{l}\text { Duvelisib (IPI-|45)+ } \\
\mathrm{B}, \mathrm{BR} \text {, or } \mathrm{R}\end{array}$ & $\begin{array}{l}\text { PI3K delta and } \\
\text { gamma }\end{array}$ & $81 \%(10 \%)$ & NA & NA \\
\hline
\end{tabular}

Abbreviations: ORR, overall response rate; CR, complete response; PFS, progression free survival; OS, overall survival; B, bendamustine; R, rituximab; BTK, Bruton's tyrosine kinase; NK cell, natural killer cell; NF- $\kappa B$, nuclear factor- $\mathrm{B}$; NA, not available; PI3K, phosphoinositide 3-kinase. 
regardless of sensitivity to prior fludarabine. The ORR among fludarabine-refractory patients was $45.5 \%$, which correlated with median duration of response of 8.7 months. Activity was noted in poor prognostic patients including del 11q (92\%) and unmutated IGVH (59\%); however, the ORR in patients with del $17 \mathrm{p}$ was only $7.1 \%$. Grade $3 / 4$ adverse events included thrombocytopenia (28\%), neutropenia (23\%), anemia (17\%), and infection (13\%).

The GCLLSG subsequently evaluated the role of BR in the front-line setting in a Phase II study of 117 patients with a median age of 64 years. ${ }^{31}$ Patients received a similar regimen; however, bendamustine was increased to $90 \mathrm{mg} / \mathrm{m}^{2}$ D1 and 2. The ORR was 88\% (CR 23\%), and the median PFS was 34 months. At a median follow-up of 27 months, the OS was $91 \%$. As in the relapsed or refractory setting, the regimen was efficacious in poor-risk patients such as del 11q 90\% (CR 40\%) and unmutated IGVH 89\% (27\%); however, the del 17 p population remained a challenge (ORR 38\%). Grade 3/4 adverse events similar to the relapsed and refractory setting are thrombocytopenia (22\%), neutropenia $(20 \%)$, anemia (20\%), allergic reaction (9\%), and infection (8\%).

The GCLLSG study found BR to be comparable to their previous results with FCR in terms of efficacy, but noted less toxicity. ${ }^{31}$ Specifically, FCR produced an ORR of $90 \%$, CR $44 \%$, and median PFS of 51.8 months. ${ }^{7}$ Grade $3 / 4$ neutropenia and infection were noted in $34 \%$ and $25 \%$ of patients, respectively. The FCR patient population was slightly younger (median age of 61 years), and a higher percentage of patients had advanced stage disease. Based on these data, the GCLLSG conducted the CLL10 study, which was an international, Phase III trial designed to assess the noninferiority of BR in comparison to FCR in previously untreated, physically fit patients with CLL. ${ }^{32}$ Patients were deemed eligible if they had a $\mathrm{CrCl}>70 \mathrm{~mL} / \mathrm{min}$, lacked del $17 \mathrm{p}$, and had a cumulative index rating score (CIRS) of $<7$. Five hundred and sixty-four patients were randomized to six, 28-day cycles of FCR (F, $25 \mathrm{mg} / \mathrm{m}^{2} \mathrm{D} 1-3$; C, $\left.250 \mathrm{mg} / \mathrm{m}^{2} \mathrm{D} 1-3 ; \mathrm{R}, 375 \mathrm{mg} / \mathrm{m}^{2} \mathrm{C} 1 \mathrm{D} 0,500 \mathrm{mg} / \mathrm{m}^{2} \mathrm{C} 2-6 \mathrm{D} 1\right)$ or $\mathrm{BR}\left(\mathrm{B}, 90 \mathrm{mg} / \mathrm{m}^{2} \mathrm{D} 1\right.$ and $2 ; \mathrm{R}, 375 \mathrm{mg} / \mathrm{m}^{2} \mathrm{C} 1 \mathrm{D} 0$, $\left.500 \mathrm{mg} / \mathrm{m}^{2} \mathrm{C} 2-6 \mathrm{D} 1\right)$. The ORR was $97.8 \%$ in both arms; however, the $\mathrm{CR}$ rate $(40.7 \%$ vs $31.5 \%, P=0.026)$ and median PFS (53.7 months vs 43.2 months, $P=0.001$ ) were higher with FCR. At 3-year follow-up, the OS was similar between both arms (91\% FCR vs $92 \%$ BR). Of note, the BR group had a significantly higher number of patients who were elderly or had unmutated IGVH status despite initial randomization. Achievement of minimal residual disease (MRD) negativity was a secondary endpoint of the study, as it has been correlated with a significantly longer event-free survival and time to next treatment. ${ }^{33}$ The rate of MRD negativity was higher in the FCR arm when evaluated by both peripheral blood and bone marrow in the available patients. FCR was associated with significantly greater grade $3 / 4$ neutropenia ( $88 \%$ vs $68 \%, P<0.001)$ and infection $(40 \%$ vs $25 \%, P=0.001)$, particularly in the elderly. Given the lack of an OS benefit, these data support the use of either FCR or BR for previously untreated patients with CLL under the age of 65 years. However, BR is preferred for the elderly based on tolerability.

\section{Bendamustine and newer anti-CD20 antibodies}

In an attempt to improve the efficacy of BR, bendamustine has been combined with several second-generation CD20 monoclonal antibodies. In comparison to the chimeric rituximab, ofatumumab is a fully human IgG anti-CD20 monoclonal antibody. Ofatumumab's membrane epitope on CD20 is more proximal than rituximab's target and encompasses both the small and large loops of CD20, theoretically resulting in higher affinity for CD20 and more effective complementdependent cytotoxicity and antibody-dependent cellular cytotoxicity (ADCC) ${ }^{34}$ Ofatumumab was initially approved for patients with fludarabine and alemtuzumab-refractory CLL based on an ORR of $58 \% .{ }^{35}$ It was combined with bendamustine in a Phase II study at the Lombardi Comprehensive Cancer Center, in which relapsed or refractory patients received ofatumumab $300 \mathrm{mg}$ C1D-7 followed by $1,000 \mathrm{mg}$ D1 and B $70 \mathrm{mg} / \mathrm{m}^{2} \mathrm{D} 1$ and 2 every 28 days for four to six cycles. ${ }^{36}$ Analysis of the first ten patients indicated an ORR of $40 \%$ (CR 20\%), median PFS of 8.1 months, and an OS of 16.2 months. The study was terminated early due to an unexpected toxicity, including severe infections, an unexplained neurotoxicity, and a higher than expected incidence of Richter's transformation ( $\mathrm{n}=3$ ). Better activity was noted in a larger Phase II study conducted by the Gruppo Italiano Malattie EMatologiche dell'Adulto [Italian Group of the Adult Hematologic Diseases], in which 47 relapsed or refractory patients treated with a similar regimen were found to have an ORR of 72\% (CR 17\%) and a 2-year PFS and OS of $50 \%$ and $84 \%$, respectively. ${ }^{37}$ Grade $3 / 4$ neutropenia occurred in $62 \%$ of patients, but did not correlate with a significant severe infection rate (2\%). Two patients developed Richter's transformation, but there were no reports of neurotoxicity as described in the previous trial. B-ofatumumab was also evaluated in a pharmaceutical-sponsored Phase II study of patients with previously untreated CLL who were deemed 
unfit for fludarabine-based chemoimmunotherapy $(n=44)$ and those with relapsed disease $(n=53) .{ }^{38}$ The response rates among the previously untreated (ORR 95\%, CR 43\%) and relapsed patients (ORR 74\%, CR 11\%) were similar to previous reports with BR. With the exception of a Phase I study of bendamustine, ofatumumab, and pentostatin in relapsed CLL and B-NHL (NCT01352312), the B-ofatumumab regimen is not actively being explored in CLL.

Obinutuzumab, a fully humanized, glycoengineered, antiCD20 antibody, has also been studied in combination with bendamustine. Unlike rituximab, which binds to the CD20 type I epitope, obinutuzumab binds with higher affinity to the extracellular domain of the CD20 type II epitope due to the glycosylation of the Fc region. ${ }^{39}$ This interaction promotes more efficacious direct cell apoptosis and ADCC, which is 35 -fold greater than rituximab. ${ }^{40}$ In 2013 , the Food and Drug Administration approved obinutuzumab in combination with Chl for previously untreated CLL based on the results of the CLL11 study. ${ }^{41}$ In this Phase III study, the GCLLSG randomized 781 patients to either Chl, rituximab, and Chl (R-Chl) or obinutuzumab and Chl (obinutuzumab-Chl). Eligible patients must have had a CIRS $>6$ and $\mathrm{CrCl}<70 \mathrm{~mL} / \mathrm{min}$. The approval was based on a significant improvement in median PFS with obinutuzumab-Chl compared to $\mathrm{Chl}$ alone (26.7 months vs 11.1 months, $P<0.001$ ). Follow-up data indicated that the combination was superior to $\mathrm{R}-\mathrm{Chl}$ as well, with a median PFS of 26.7 months vs 15.2 months $(P<0.001)$; however, a higher rate of grade $3 / 4$ infusionrelated reactions was noted $(20 \%$ vs $4 \%$ ). The GALTON trial explored effectiveness of obinutuzumab with more aggressive chemotherapy regimens. ${ }^{42}$ In this Phase Ib study, obinutuzumab (100 mg C1D1, $900 \mathrm{mg} \mathrm{C1D2,} \mathrm{1,000} \mathrm{mg}$ C1D8 and 5, C2-6D1) was administered with either FC or $\mathrm{B}$ in 41 treatment-naïve patients. The ORR was surprisingly lower with FC-obinutuzumab (62\%, CR 10\%) than with B-obinutuzumab (90\%, CR 20\%). The most common grade $3 / 4$ adverse events in the FC-obinutuzumab and B-obinutuzumab arms were neutropenia $(29 \%, 50 \%)$, infusion-related reactions $(29 \%, 10 \%)$, febrile neutropenia $(19 \%, 10 \%)$, and hepatic transaminitis $(19 \%, 10 \%)$. Obinutuzumab is currently being investigated with bendamustine in a series of pharmaceutical sponsored studies in patients with previously untreated CLL (NCT02320487), relapsed and refractory CLL (NCT02071225), or both (NCT01905943). The GCLLSG is evaluating the use of obinutuzumab as a maintenance therapy following induction with B-obinutuzumab or FC-obinutuzumab in relapsed and refractory CLL (NCT02320383).

\section{Bendamustine and other monoclonal antibodies}

Combinations with antibodies to alternative targets have also been explored. Alemtuzumab, a humanized anti-CD52 monoclonal antibody approved as a single agent for CLL, was one of the first to be combined with bendamustine. ${ }^{43,44}$ While promising results of an ORR of $68 \%$ (CR 24\%) and median PFS of 17.3 months were seen in a Phase I/II study of relapsed and refractory disease, the regimen was associated with considerable infectious toxicity, including symptomatic cytomegalovirus reactivation. ${ }^{45}$ Furthermore, alemtuzumab is no longer commercially available as an anticancer therapy in the USA. Other antibodies have been investigated including MEDI-551, a humanized, glycosylated anti-CD19 monoclonal antibody, and otlertuzumab (TRU-016), a humanized IgG protein against CD37. Both have shown modest activity as single agents in relapsed CLL. ${ }^{46,47}$ Early phase studies evaluating combinations with bendamustine have indicated ORRs of $48 \%-64 \%$ and $69 \%$ in relapsed and refractory patients, respectively; however, further development in CLL is unclear. ${ }^{48,49}$

\section{$B R$ and other chemotherapeutic agents}

The addition of conventional chemotherapeutic agents to BR has been explored in early phase clinical trials. Mitoxantrone and cytarabine have been combined with BR, producing ORRs of $84 \%-86 \%$ (CR 27\%-38\%) with relatively short median PFSs (10-16 months). ${ }^{50,51}$ Both regimens were limited by hematologic toxicity and infection. As these types of regimens have thus far failed to show a significant improvement in efficacy compared to BR, investigative interests have shifted toward biologic therapies, which exploit tumor specific pathways as well as the tumor microenvironment. Remarkable efficacy and a relatively mild adverse event profile have enabled bendamustine to be an ideal agent for combination with these novel therapies.

\section{Bendamustine and immunomodulatory agents}

Lenalidomide, a modulator of the tumor microenvironment, has multiple mechanisms of action including downregulation of prosurvival cytokines (TNF- $\alpha$, IL-6, IL-8) and activation of T- and NK cells. ${ }^{52}$ The Roswell Park Cancer Institute first explored the use of lenalidomide in CLL in a Phase II study of 45 patients with relapsed or refractory disease, reporting an ORR of 47\% (CR 9\%)..$^{53}$ These initial data launched several studies in CLL. The Lombardi Comprehensive Cancer Center conducted a Phase I study in which patients with 
relapsed or refractory disease received B $90 \mathrm{mg} / \mathrm{m}^{2} \mathrm{D} 1$ and 2 and escalating doses of lenalidomide (5-20 mg) for up to six, 28-day cycles. ${ }^{54}$ Among the seven evaluable patients, the ORR was 57\% (CR 14\%), and median PFS was 14 months. Investigators at Massachusetts General Hospital and Dana Farber Cancer Institute explored the triplet in a Phase I study of previously untreated patients. ${ }^{55}$ Of the 23 patients enrolled, the ORR was $87 \%$ with a $\mathrm{CR} / \mathrm{CR}$ incomplete of $39 \%$. An ORR of $92 \%$ was seen at the maximum tolerated dose (MTD) (B, $90 \mathrm{mg} / \mathrm{m}^{2} \mathrm{D} 1$ and 2; lenalidomide, $5 \mathrm{mg}$ C1D8-21, C2-6, 10 mg D1-21); however, significant dose reduction was required in subsequent cycles due to toxicity including neutropenia and rash.

\section{Bendamustine and small molecule inhibitors}

A greater understanding of the role of the B-cell receptor (BCR) in the pathogenesis of CLL has ushered in a new era of anticancer therapy. Several oral small molecule inhibitors have been developed against aberrantly amplified signaling pathways downstream of the BCR such as Bruton's tyrosine kinase (BTK) and phosphoinositide 3-kinase (PI3K). ${ }^{56}$ Inhibition of these and similar targets has resulted in impressive response rates, particularly for those with high-risk disease.

Ibrutinib, a strong, irreversible inhibitor of BTK, was granted accelerated approval for relapsed and refractory CLL based on a Phase Ib/II trial demonstrating an ORR of $71 \%$ and a 26 -month PFS of $75 \% .^{57}$ The RESONATE study secured this approval by demonstrating a significant improvement in median PFS (not reached vs 8.1 months, $P<0.001)$ and 1 -year OS (90\% vs $81 \%, P=0.005)$ compared to ofatumumab. ${ }^{58}$ Based on the these results, the Alliance for Clinical Trials in Oncology is challenging the front-line role of BR in elderly patients in an ongoing Phase III comparison of BR vs ibrutinib vs R-ibrutinib (NCT01886872). Encouraging early phase data of BR-Ibrutinib in the relapsed and refractory setting have prompted the Phase III HELIOS trial in which 578 previously treated patients were randomized to receive $\mathrm{BR}\left(\mathrm{B}, 70 \mathrm{mg} / \mathrm{m}^{2} ; \mathrm{R}, 375 \mathrm{mg} / \mathrm{m}^{2} ; \mathrm{C} 1\right.$, $500 \mathrm{mg} / \mathrm{m}^{2} \mathrm{C} 2-6$ ) with ibrutinib (420 mg daily) followed by ibrutinib maintenance or BR and placebo. ${ }^{59,60}$ The ORR (CR) was $83 \%(10 \%)$ with the triplet vs $68 \%(3 \%), P<0.0001$. At a median follow-up of 17 months, the median PFS had not been reached with BR-Ibrutinib in comparison to 13 months with BR $(P<0.0001$, HR 0.20). The median OS had not been reached in either arm, but was confounded by the allowance of crossover. The adverse events were consistent with the known toxicity profiles of the agents. These are the first Phase III data to support the use of a small molecule kinase inhibitor with BR.

Idelalisib, a potent inhibitor of the delta isoform of PI3K, was approved for combination with rituximab in relapsed or refractory CLL based on a superior ORR (81\% vs 13\%) and median PFS (not reached vs 5.5 months) compared to rituximab $(P<0.001) .{ }^{61}$ Idelalisib was studied in combination with B and BR as part of a three-arm Phase I trial in heavily pretreated CLL (median of three prior therapies). ${ }^{62}$ Fifty-two patients received idelalisib $150 \mathrm{mg}$ twice daily continuously with either B (70 mg/m² or $90 \mathrm{mg} / \mathrm{m}^{2} \mathrm{C} 1-6 \mathrm{D} 1$ and 2$), \mathrm{R}$ (375 mg/m² weekly $\times 8$ doses), or BR for six, 28-day cycles. The ORR was $81 \%$ (CR 2\%), and the 2-year PFS and OS were $62 \%$ and $85 \%$, respectively. Nonhematologic grade $3 / 4$ adverse events included diarrhea/colitis (14\%), pneumonia $(12 \%)$, and transaminitis $(10 \%)$. These data prompted two Phase III, randomized, double-blind trials investigating idelalisib in combination with BR in recurrent and previously untreated CLL, both of which have completed accrual and results are pending (NCT01569295, NCT01980888). Duvelisib (IPI-145), a second-generation selective inhibitor of the delta and gamma isoforms of PI3K, has also demonstrated promising activity in CLL. In a Phase I study, duvelisib produced an ORR of 55\% (CR 2\%) in 48 patients with relapsed or refractory disease. ${ }^{63}$ It is currently being investigated in combination with $\mathrm{B}, \mathrm{R}$, and $\mathrm{BR}$ in an ongoing Phase Ib study of relapsed and refractory B-cell malignancies. ${ }^{64}$ An interim analysis of 21 patients from all arms revealed an ORR of 81\% (CR 10\%); however, data from the individual arms and histologic subtypes are not available at this time. Grade 3/4 adverse events were neutropenia (28\%), transaminitis (18\%), and rash (16\%). Combinations with novel agents targeting other components of the BCR signaling cascade, such as MK2206, a potent Akt inhibitor, everolimus, a mammalian target of rapamycin (mTOR) inhibitor and SAR245409, a selective oral inhibitor of PI3K and mTOR, are ongoing (NCT01369849, NCT02240719, NCT01410513).

One of the most exciting intracellular B-cell targets distinct from the BCR is the BCL-2 family of proteins. Comprised of pro- and antiapoptotic members, these molecules mediate apoptosis at the mitochondrial level. ${ }^{65} \mathrm{BCL}-2$ is overexpressed in CLL and is a critical component in the pathogenesis of the disease. ${ }^{66}$ Preclinical studies indicate that inhibition of the antiapoptotic members allows for the release of the sequestered proapoptotic counterparts and subsequent programed cell death. ${ }^{67}$ Venetoclax (ABT-199) is a second-generation, highly potent selective inhibitor of 
BCL-2, reengineered to overcome the dose-limiting thrombocytopenia of its predecessor, navitoclax. In a Phase I study of 84 patients with relapsed or refractory CLL, venetoclax produced an ORR of $79 \%$ (CR 22\%) and median PFS of 20.5 months. ${ }^{68}$ As with BCR antagonists, remarkable activity was demonstrated in patients with del 17p (ORR 78\%) and fludarabine-refractory disease (ORR 79\%). In a preliminary safety analysis of a Phase Ib study of venetoclax and BR ( $n=6)$, grade $3 / 4$ adverse events included neutropenia $(50 \%)$, anemia (33\%), thrombocytopenia (33\%), hypertension (17\%), and pulmonary embolism (17\%). ${ }^{69}$ Efficacy data are not yet available. The GCLLSG is conducting a Phase II study, CLL2-BAG, in order to evaluate the sequential regimen of bendamustine debulking followed by venetoclax and obinutuzumab in previously treated and treatment-naive CLL (NCT02401503). In addition, venetoclax is being compared to BR in a Phase III study of R-venetoclax vs BR in relapsed and refractory CLL (NCT02005471).

\section{Safety}

As many of these studies have illustrated, bendamustine possesses an acceptable safety profile that allows for administration to older patients and those with confounding medical diagnoses, including renal and hepatic failure. Furthermore, bendamustine can be safely combined with other biologic therapies. Mild nausea and vomiting can occur, but are typically alleviated with $5 \mathrm{HT} 3$-antagonists. ${ }^{70}$ The most notable grade $3 / 4$ adverse effect is neutropenia; however, the use of prophylactic growth factor support or antibiotics is unnecessary due to a low risk of infection. Cutaneous adverse events include rash and infusion-site reactions. ${ }^{11}$ Steven-Johnson's syndrome and toxic epidermal necrolysis are less common and typically occur with concurrent allopurinol administration. Neurotoxicity has been rarely reported with administration. ${ }^{36,71}$ Secondary malignancies including myelodysplastic syndrome (MDS), acute myeloid leukemia (AML), myeloproliferative neoplasms, colorectal cancer, bronchial carcinoma, and squamous cell carcinoma have been reported in patients who have previously received bendamustine. ${ }^{11}$ Prior exposure to an alkylating agent is theorized to be a contributing factor to the development of MDS and AML. ${ }^{72-74}$ Unlike fludarabine, bendamustine does not potentiate autoimmune hemolytic anemia.

\section{Dosing and schedule}

Due to the variation in the use of bendamustine in early trials, an international consensus panel was held in order to standardize its administration for different hematological malignancies. ${ }^{70} \mathrm{~A}$ dose reduction is recommended for use with concurrent rituximab due to the risk of myelotoxicity. Similarly, the recommended dose in patients with prior fludarabine exposure is lower with the given poorer bone marrow reserve. Ideally, six cycles should be given to patients with treatment-naïve CLL, and four to six cycles can be used for individuals with heavily pretreated disease.

\section{Conclusion}

The role of bendamustine has advanced considerably since its initial approval for the treatment of CLL. Its bifunctional features and favorable toxicity profile allow for effective administration to the elderly and patients with multiple comorbidities. As bendamustine is one of the only cytotoxic chemotherapeutic agents actively undergoing clinical investigation in the lymphoid malignancies, its place in the treatment algorithm for CLL continues to evolve. Based on the results of the CLL10 study, BR is now considered an acceptable front-line option for younger, physically fit patients with previously untreated disease. The focus of current Phase III research efforts has shifted toward understanding the benefit of combining BR with small molecule inhibitors, which have distinct activity in patients with traditionally high-risk disease. In addition, ongoing trials with these novel agents are challenging the use of bendamustine as in the alliance front-line trial of BR vs ibrutinib vs R-ibrutinib and the pharmaceutical sponsored study of BR vs R-venetoclax in relapsed and refractory disease. In conjunction with these studies, there is a strong need for sound correlative analyses evaluating for predictive biomarkers and mutations of resistance. The acquisition of these types of data will hopefully enable us to tailor efficacious regimens to individual patients, thus improving long-term outcomes.

\section{Disclosure}

The authors report no conflicts of interest in this work.

\section{References}

1. Siegel RL, Miller KD, Jemal A. Cancer statistics, 2015. CA Cancer J Clin. 2015;65(1):5-29.

2. Rai KR, Sawitsky A, Cronkite EP, Chanana AD, Levy RN, Pasternack BS. Clinical staging of chronic lymphocytic leukemia Blood. 1975;46(2):219-234.

3. Binet JL, Auquier A, Dighiero G, et al. A new prognostic classification of chronic lymphocytic leukemia derived from a multivariate survival analysis. Cancer. 1981;48(1):198-206.

4. Hallek M, Cheson BD, Catovsky D, et al; International Workshop on Chronic Lymphocytic Leukemia. Guidelines for the diagnosis and treatment of chronic lymphocytic leukemia: a report from the international workshop on chronic lymphocytic leukemia updating the national cancer institute-working group 1996 guidelines. Blood. 2008;111(12) $5446-5456$. 
5. Byrd JC, Peterson BL, Morrison VA, et al. Randomized phase 2 study of fludarabine with concurrent versus sequential treatment with rituximab in symptomatic, untreated patients with B-cell chronic lymphocytic leukemia: results from cancer and leukemia group B 9712 (CALGB 9712). Blood. 2003;101(1):6-14.

6. Keating MJ, O'Brien S, Albitar M, et al. Early results of a chemoimmunotherapy regimen of fludarabine, cyclophosphamide, and rituximab as initial therapy for chronic lymphocytic leukemia. J Clin Oncol. 2005;23(18):4079-4088.

7. Hallek M, Fischer K, Fingerle-Rowson G, et al; International Group of Investigators, German Chronic Lymphocytic Leukaemia Study Group. Addition of rituximab to fludarabine and cyclophosphamide in patients with chronic lymphocytic leukaemia: a randomised, open-label, phase 3 trial. Lancet. 2010;376(9747):1164-1174.

8. Ozegowski W, Krebs D. IMET 3393, (-[1-methyl-5-bis-( $\beta$-chloroethyl)amino-benzimidazolyl-(2)]-butyric acid hydrochloride, a new cytostatic agent from among the series of benzimidazole mustard compounds. Zbl Pharm. 1971;110:1013-1019.

9. Leoni LM, Bailey B, Reifert J, et al. Bendamustine (treanda) displays a distinct pattern of cytotoxicity and unique mechanistic features compared with other alkylating agents. Clin Cancer Res. 2008;14(1):309-317.

10. Strumberg D, Harstrick A, Doll K, Hoffmann B, Seeber S. Bendamustine hydrochloride activity against doxorubicin-resistant human breast carcinoma cell lines. Anticancer Drugs. 1996;7(4):415-421.

11. Cephalon I. Treanda (bendamustine hydrochloride for injection) for intravenous infusion [US prescribing information]; 2008.

12. Matthias M, Preiss R, Sohr R, Possinger K. Pharmacokinetics of bendamustine in patients with malignant tumors. Proc Am Soc Clin Oncol. $1995 ; 14: 458$.

13. Teichert J, Baumann F, Chao Q, et al. Characterization of two phase I metabolites of bendamustine in human liver microsomes and in cancer patients treated with bendamustine hydrochloride. Cancer Chemother Pharmacol. 2007;59(6):759-770.

14. Teichert J, Sohr R, Baumann F, et al. Synthesis and characterization of some new phase II metabolites of the alkylator bendamustine and their identification in human bile, urine, and plasma from patients with cholangiocarcinoma. Drug Metab Dispos. 2005;33(7): 984-992.

15. Owen JS, Melhem M, Passarell JA, D’Andrea D, Darwish M, Kahl B. Bendamustine pharmacokinetic profile and exposure-response relationships in patients with indolent non-Hodgkin's lymphoma. Cancer Chemother Pharmacol. 2010;66(6):1039-1049.

16. Weide R. Bendamustine HCL for the treatment of relapsed indolent non-Hodgkin's lymphoma. Ther Clin Risk Manag. 2008;4(4): 727-732.

17. Shoji J, Lew SQ. The use of rituximab and bendamustine in treating chronic lymphocytic leukaemia (CLL) in end-stage renal disease (ESRD). BMJ Case Rep. 2013;2013.

18. McCloskey JK, Broome CM, Cheson BD. Safe and effective treatment of aggressive non-Hodgkin lymphoma with rituximab and bendamustine in patients with severe liver impairment. Clin Adv Hematol Oncol. 2013; 11(3):184-188.

19. Ogura M. Rituximab plus bendamustine for the treatment of aggressive non-Hodgkin lymphoma patients with severe liver impairment. Clin Adv Hematol Oncol. 2013;11(3):188-189.

20. Lissitchkov T, Arnaudov G, Peytchev D, Merkle K. Phase-I/II study to evaluate dose limiting toxicity, maximum tolerated dose, and tolerability of bendamustine $\mathrm{HCl}$ in pre-treated patients with B-chronic lymphocytic leukaemia (binet stages B and C) requiring therapy. J Cancer Res Clin Oncol. 2006;132(2):99-104.

21. Kath R, Blumenstengel K, Fricke HJ, Hoffken K. Bendamustine monotherapy in advanced and refractory chronic lymphocytic leukemia. $J$ Cancer Res Clin Oncol. 2001;127(1):48-54.

22. Aivado M, Schulte K, Henze L, Burger J, Finke J, Haas R. Bendamustine in the treatment of chronic lymphocytic leukemia: results and future perspectives. Semin Oncol. 2002;29(4 Suppl 13):19-22.
23. Bremer K. High rates of long-lasting remissions after 5-day bendamustine chemotherapy cycles in pre-treated low-grade non-Hodgkin'slymphomas. J Cancer Res Clin Oncol. 2002;128(11):603-609.

24. Bergmann MA, Goebeler ME, Herold M, et al; German CLL Study Group. Efficacy of bendamustine in patients with relapsed or refractory chronic lymphocytic leukemia: results of a phase I/II study of the German CLL study group. Haematologica. 2005;90(10):1357-1364.

25. Niederle N, Megdenberg D, Balleisen L, et al. Bendamustine compared to fludarabine as second-line treatment in chronic lymphocytic leukemia. Ann Hematol. 2013;92(5):653-660.

26. Knauf WU, Lissichkov T, Aldaoud A, et al. Phase III randomized study of bendamustine compared with chlorambucil in previously untreated patients with chronic lymphocytic leukemia. J Clin Oncol. 2009;27(26):4378-4384.

27. Knauf WU, Lissitchkov T, Aldaoud A, et al. Bendamustine compared with chlorambucil in previously untreated patients with chronic lymphocytic leukaemia: updated results of a randomized phase III trial. $\mathrm{Br}$ J Haematol. 2012;159(1):67-77.

28. Chow KU, Sommerlad WD, Boehrer S, et al. Anti-CD20 antibody (IDEC-C2B8, rituximab) enhances efficacy of cytotoxic drugs on neoplastic lymphocytes in vitro: role of cytokines, complement, and caspases. Haematologica. 2002;87(1):33-43.

29. Rummel MJ, Al-Batran SE, Kim SZ, et al. Bendamustine plus rituximab is effective and has a favorable toxicity profile in the treatment of mantle cell and low-grade non-Hodgkin's lymphoma. J Clin Oncol. 2005;23(15):3383-3389.

30. Fischer K, Cramer P, Busch R, et al. Bendamustine combined with rituximab in patients with relapsed and/or refractory chronic lymphocytic leukemia: a multicenter phase II trial of the German chronic lymphocytic leukemia study group. J Clin Oncol. 2011;29(26):3559-3566.

31. Fischer K, Cramer P, Busch R, et al. Bendamustine in combination with rituximab for previously untreated patients with chronic lymphocytic leukemia: a multicenter phase II trial of the German chronic lymphocytic leukemia study group. J Clin Oncol. 2012;30(26):3209-3216.

32. Eichhorst B, Fink AM, Busch R, et al. Frontline chemoimmunotherapy with fludarabine (F), cyclophosphamide (C), and rituximab (R) (FCR) shows superior efficacy in comparison to bendamustine (B) and rituximab (BR) in previously untreated and physically fit patients (pts) with advanced chronic lymphocytic leukemia (CLL): final analysis of an international, randomized study of the German CLL study group (GCLLSG) (CLL10 study). Blood. 2014. [abstract 19; ASH Annual Meeting Abstracts].

33. Farren TW, Giustiniani J, Fanous M, et al. Minimal residual disease detection with tumor-specific CD160 correlates with event-free survival in chronic lymphocytic leukemia. Blood Cancer J. 2015;5:e273.

34. Lin TS. Ofatumumab: a novel monoclonal anti-CD20 antibody. Pharmgenomics Pers Med. 2010;3:51-59.

35. Wierda WG, Kipps TJ, Mayer J, et al. Ofatumumab as single-agent CD20 immunotherapy in fludarabine-refractory chronic lymphocytic leukemia. J Clin Oncol. 2010;28(10):1749-1755.

36. Ujjani C, Ramzi P, Gehan E, Wang H, Wang Y, Cheson BD. Ofatumumab and bendamustine in previously treated chronic lymphocytic leukemia and small lymphocytic lymphoma. Leuk Lymphoma. 2014;55:1-6.

37. Cortelezzi A, Sciumè M, Liberati AM, et al. Bendamustine in combination with ofatumumab in relapsed or refractory chronic lymphocytic leukemia: a GIMEMA multicenter phase II trial. Leukemia. 2014;28(3): 642-648.

38. Offner F, Panagiotidis P, Afanasyev B, et al. Ofatumumab and bendamustine combination therapy in patients with untreated and relapsed chronic lymphocytic leukemia: initial results of the phase II study OMB115991. In: XV iwCLL; 2013. Abstract 4.29.

39. Robak T. GA-101, a third-generation, humanized and glyco-engineered anti-CD20 mAb for the treatment of B-cell lymphoid malignancies. Curr Opin Investig Drugs. 2009;10(6):588-596.

40. Mössner E, Brünker P, Moser S, et al. Increasing the efficacy of CD20 antibody therapy through the engineering of a new type II anti-CD20 antibody with enhanced direct and immune effector cell-mediated B-cell cytotoxicity. Blood. 2010;115(22):4393-4402. 
41. Goede V, Fischer K, Busch R, et al. Obinutuzumab plus chlorambucil in patients with CLL and coexisting conditions. $N$ Engl J Med. 2014;370(12):1101-1110.

42. Brown JR, O'Brien S, Kingsley CD, et al. Obinutuzumab plus fludarabine/cyclophosphamide or bendamustine in the initial therapy of CLL patients: the phase 1b GALTON trial. Blood. 2015;125(18): 2779-2785.

43. Keating MJ, Flinn I, Jain V, et al. Therapeutic role of alemtuzumab (campath-1H) in patients who have failed fludarabine: results of a large international study. Blood. 2002;99(10):3554-3561.

44. Hillmen P, Skotnicki AB, Robak T, et al. Alemtuzumab compared with chlorambucil as first-line therapy for chronic lymphocytic leukemia. J Clin Oncol. 2007;25(35):5616-5623.

45. Montillo M, Tedeschi A, Gaidano G, et al. Bendamustine and subcutaneous alemtuzumab combination is an effective treatment in relapsed/ refractory chronic lymphocytic leukemia patients. Haematologica. 2014;99(9):e159-e161.

46. Hamadani M, Forero A, Kipps TJ, et al. MEDI-551, an anti-CD19 antibody active in chronic lymphocytic leukemia (CLL) patients previously treated with rituximab. J Clin Oncol. 2013. [abstract 7045; ASCO Annual Meetings].

47. Byrd JC, Pagel JM, Awan FT, et al. A phase 1 study evaluating the safety and tolerability of otlertuzumab, an anti-CD37 mono-specific ADAPTIR therapeutic protein in chronic lymphocytic leukemia. Blood. 2014;123(9):1302-1308.

48. Gladstone D, Andre M, Zaucha J, et al. Phase 2 open-label study of MEDI-551 and bendamustine versus rituximab and bendamustine in adults with relapsed or refractory CLL. J Clin Oncol. 2014. [abstract 3028; ASCO Annual Meetings].

49. Robak T, Hellman A, Loscertales J, et al. Phase 2 study of otlertuzumab (TRU-016), an anti-CD37 ADAPTIR ${ }^{\mathrm{TM}}$ protein, in combination with bendamustine vs bendamustine alone in patients with relapsed chronic lymphocytic leukemia (CLL) - updated results. Blood. 2014. [abstract 5642; ASH Annual Meeting Abstracts].

50. Weide R, Pandorf A, Heymanns J, Koppler H. Bendamustine/ mitoxantrone/rituximab (BMR): a very effective, well tolerated outpatient chemoimmunotherapy for relapsed and refractory CD20-positive indolent malignancies. final results of a pilot study. Leuk Lymphoma. 2004;45(12):2445-2449.

51. Visco C, Finotto S, Pomponi F, et al. The combination of rituximab, bendamustine, and cytarabine for heavily pretreated relapsed/refractory cytogenetically high-risk patients with chronic lymphocytic leukemia. Am J Hematol. 2013;88(4):289-293.

52. Chanan-Khan A, Cheson BD. Lenalidomide for the treatment of B-cell malignancies. J Clin Oncol. 2008;26(9):1544-1552.

53. Chanan-Khan A, Miller KC, Musial L, et al. Clinical efficacy of lenalidomide in patients with relapsed or refractory chronic lymphocytic leukemia: results of a phase II study. J Clin Oncol. 2006;24(34):5343-5349.

54. Ujjani CS, Karim SM, Goswami T, et al. Bendamustine and lenalidomide in relapsed/refractory CLL. Blood. 2011. [abstract 4618; ASH Annual Meeting Abstracts].

55. Abramson J, Davids M, Werner L, et al. Lenalidomide added to bendamustine-rituximab for untreated chronic lymphocytic leukemia (CLL): a phase 1 study. J Clin Oncol. 2015. [abstract 7082; ASCO Annual Meetings].

56. Woyach JA, Johnson AJ, Byrd JC. The B-cell receptor signaling pathway as a therapeutic target in CLL. Blood. 2012;120(6):1175-1184.

57. Byrd JC, Furman RR, Coutre SE, et al. Targeting BTK with ibrutinib in relapsed chronic lymphocytic leukemia. $N$ Engl J Med. 2013;369(1):32-42.

58. Byrd JC, Brown JR, O'Brien S, et al; RESONATE Investigators. Ibrutinib versus ofatumumab in previously treated chronic lymphoid leukemia. N Engl J Med. 2014;371(3):213-223.
59. Brown J, Barrientos J, Barr P, et al. Ibrutinib in combination with bendamustine and rituximab is active and tolerable in patients with relapsed/refractory CLL/SLL: final results of a phase $1 \mathrm{~b}$ study. Blood. 2013. [abstract 525; ASH Annual Meeting Abstracts].

60. Chanan-Khan A, Cramer P, Demirkan F, et al. Ibrutinib combined with bendamustine and rituximab in previously treated chronic lymphocytic leukemia/small lymphocytic lymphoma: first results from a randomized, double-blind, placebo-controlled, phase III study. J Clin Oncol. 2015. [abstract LBA7005; ASCO Annual Meetings].

61. Furman RR, Sharman JP, Coutre SE, et al. Idelalisib and rituximab in relapsed chronic lymphocytic leukemia. N Engl J Med. 2014;370(11): 997-1007.

62. Barrientos JC, Furman RR, Lenoard J, et al. Update on a phase I study of the selective PI3K $\delta$ inhibitor idelalisib (GS-1101) in combination with rituximab and/or bendamustine in patients with relapsed or refractory CLL. J Clin Oncol. 2013. [abstract 7017; ASCO Annual Meetings].

63. O'Brien S, Patel M, Kahl BS, et al. Duvelisib (IPI-145), a PI3K- $\delta, \gamma$ inhibitor, is clinically active in patients with relapsed/refractory chronic lymphocytic leukemia. Blood. 2014. [abstract 3334; ASH Annual Meeting Abstracts].

64. Flinn I, Patel MR, Maris MB, Matous J, Cherry M, Berdeja JG. An open-label, phase Ib study of duvelisib (IPI-145) in combination with bendamustine, rituximab or bendamustine/rituximab in select subjects with lymphoma or chronic lymphocytic leukemia. Blood. 2014. [abstract 4422; ASH Annual Meeting Abstracts].

65. Cory S, Adams JM. The Bc12 family: regulators of the cellular life-ordeath switch. Nat Rev Cancer. 2002;2(9):647-656.

66. Letai A. A new face of BCL-2 inhibition in CLL. Blood. 2011;117(10): 2750-2751.

67. Del Gaizo Moore V, Brown JR, Certo M, Love TM, Novina CD, Letai A. Chronic lymphocytic leukemia requires BCL2 to sequester prodeath BIM, explaining sensitivity to BCL2 antagonist ABT-737. $J$ Clin Invest. 2007;117(1):112-121.

68. Seymour J, Davids M, Pagel J, et al. ABT-199 (GDC-0199) in relapsed/ refractory $(\mathrm{R} / \mathrm{R})$ chronic lymphocytic leukemia (CLL) and small lymphocytic lymphoma (SLL): high complete- response rate and durable disease control. J Clin Oncol. 2014. [abstract 7015; ASCO Annual Meetings].

69. Salles GA, Boyd TE, Morschhauser F, et al. Preliminary results of a phase 1b study (GO28440) combining GDC-0199 (ABT-199) with bendamustine/rituximab in patients with relapsed/refractory or previously untreated chronic lymphocytic leukemia. Blood. 2014. [abstract 3337; ASH Annual Meeting Abstracts].

70. Cheson BD, Wendtner CM, Pieper A, et al. Optimal use of bendamustine in chronic lymphocytic leukemia, non-Hodgkin lymphomas, and multiple myeloma: treatment recommendations from an international consensus panel. Clin Lymphoma Myeloma Leuk. 2010;10(1):21-27.

71. Cheson BD, Kroll ML. Bendamustine induced neurotoxicity. Clin Adv Hematol Oncol. 2009;7(11):743-746.

72. Herold M, Schulze A, Niederwieser D, et al; East German Study Group Hematology and Oncology (OSHO). Bendamustine, vincristine and prednisone (BOP) versus cyclophosphamide, vincristine and prednisone (COP) in advanced indolent non-Hodgkin's lymphoma and mantle cell lymphoma: results of a randomised phase III trial (OSHO\# 19). J Cancer Res Clin Oncol. 2006;132(2):105-112.

73. Friedberg JW, Cohen P, Chen L, et al. Bendamustine in patients with rituximab-refractory indolent and transformed non-Hodgkin's lymphoma: results from a phase II multicenter, single-agent study. J Clin Oncol. 2008;26(2):204-210.

74. Kahl BS, Bartlett NL, Leonard JP, et al. Bendamustine is effective therapy in patients with rituximab-refractory, indolent B-cell non-Hodgkin lymphoma: results from a multicenter study. Cancer. 2010;116(1):106-114 


\section{Publish your work in this journal}

Blood and Lymphatic Cancer: Targets and Therapy is an international, peer-reviewed, open access journal focusing on blood and lymphatic cancer research, identification of therapeutic targets and the optimal use of preventative and integrated treatment interventions to achieve improved outcomes, enhanced survival and quality of life for the

cancer patient. The manuscript management system is completely online and includes a very quick and fair peer-review system. Visit http://www.dovepress.com/testimonials.php to read real quotes from published authors.

Submit your manuscript here: http://www.dovepress.com/blood-and-lymphatic-cancer-targets-and-therapy-journal 